\title{
Tangence
}

\section{Fictions anciennes et modernes du politique}

\section{François Dugré}

Numéro 63, juin 2000

Fictions et politique

URI : https://id.erudit.org/iderudit/008181ar

DOI : https://doi.org/10.7202/008181ar

Aller au sommaire du numéro

Éditeur(s)

Presses de l'Université du Québec

ISSN

0226-9554 (imprimé)

1710-0305 (numérique)

Découvrir la revue

Citer cet article

Dugré, F. (2000). Fictions anciennes et modernes du politique. Tangence, (63), 28-54. https://doi.org/10.7202/008181ar d'utilisation que vous pouvez consulter en ligne.

https://apropos.erudit.org/fr/usagers/politique-dutilisation/ 


\section{Fictions anciennes et modernes du politique François Dugré, Collège François-Xavier-Garneau}

Si on confond l'État avec la société civile et si on le destine à la sécurité et à la protection de la propriété et de la liberté personnelles, l'intérêt des individus en tant que tels est le but suprême en vue duquel ils sont rassemblés et il en résulte qu'il est facultatif d'être membre d'un État.

Hegel, Principes de la philosophie du droit, $₫ 258$

Il me faut avouer d'entrée de jeu que la thématique "fictions du politique" ouvre un champ si vaste de possibles que j'ai hésité entre plusieurs projets d'articles. Or, il me semble qu'un des problèmes les plus aigus auxquels nous ayons à faire face - et donc un des plus pertinents à méditer - est celui qu'il faut bien nommer la fictionalisation croissante du politique face à divers mouvements centripètes qui tendent tous à excentrer et à fragiliser la capacité d'autonomie politique de la société civile, à miner l'idéal républicain et l'humanisme civique. Certes, je songe notamment à l'atomisation sociale, à l'individualisme, et au despotisme doux qu'anticipait et redoutait déjà Tocqueville. De même, l'épigraphe de cet article témoigne de la volonté hégélienne de contrer l'éthique utilitaire des Lumières, son individualisme homogénéisant qui, appuyé sur l'universalisme abstrait des droits individuels, fait perdre aux diverses communautés leurs traits particuliers; cette "furie destructrice" des sentiments d'appartenance laisse peu de place au foyer identitaire que favorisait la vie civique et tend inévitablement vers une sorte de cosmopolitisme niais ${ }^{1}$. Mais si ces divers problèmes demeurent bien évidemment les nôtres — comment harmoniser l'universalité des principes de nos constitutions

1. Grand démocrate égalitariste, Rousseau fut néanmoins sensible à ce problème: "Il n'y a plus aujourd'hui de François, d'Allemands, d'Espagnols, d'Anglois même, quoiqu'on en dise; il n'y a que des Européens. Tous ont les mêmes goûts, les mêmes passions, les mêmes mœurs, parce que aucun n'a reçu de forme nationale par une institution particulière." Considérations sur le gouvernement de Pologne, dans Euvres complètes, tome III, Paris, Gallimard, 1964, p. 960. 
libérales avec la diversité vivante des communautés historiques, comment réconcilier la Moralität et la Sittlichkeit ${ }^{2}$ ? - , je songe avant tout à deux phénomènes plus contemporains qui assurément prolongent, exacerbent et accusent ces divers "malaises" modernes. Si l'on évoque fréquemment la tendance lourde de la judiciarisation du politique court-circuitant et se substituant trop souvent au débat démocratique afin de régler les litiges ${ }^{3}$, on semble encore sous-estimer les dangers potentiels et réels du modèle libéral de la primauté du droit qui se traduit par l'empiètement de l'autorité judiciaire sur le pouvoir législatif, livrant ainsi les citoyens au statut de spectateurs de décisions prises par d'autres qu'eux-mêmes et qui affectent leur vie. Ce juridisme envahissant traduit et encourage d'ailleurs l'illusion de résoudre par le langage des droits l'ensemble des dilemmes éthiques et politiques contemporains tout en confortant son corollaire immédiat, l'instrumentalisation d'un État neutre, "veilleur de nuit": "aucun État plus étendu que l'État minimal ne peut-être justifié ${ }^{4}$ ". Je songe ensuite à la mondialisation des marchés et à l'extraordinaire influence, sans précédent, des marchés financiers qui déterminent désormais largement la valeur des monnaies des divers pays, leurs (?) politiques économiques et sociales, sans même parler des publicités et des "produits culturels" standardisés qui débilitent et "théâtralisent" toujours davantage la vie sociale. "There is no such a thing as a society ", disait $M^{\text {me }}$ Thatcher, à quoi M. Bouchard fit écho, à sa manière, lors du sommet socioéconomique de 1996 devant les représentants des principaux groupes d'intérêts du Québec: "les véritables décideurs ne sont pas ici". Les protestations contre l'Accord multilatéral sur l'investissement négocié en secret, de même que celles, plus médiatisées, lors de la conférence de l'Organisation mondiale du commerce à Seattle, auront favorisé une prise de conscience plus large du déficit démocratique de ces "manœuvres". Malgré son catastrophisme et

2. J. Habermas, L'intégration républicaine. Essais de théorie politique, traduction de R. Rochlitz, Paris, Fayard, 1998, p. 280 : "À vrai dire, la philosophie politique n'a jamais sérieusement réussi à surmonter la tension entre souveraineté du peuple et droits de l'homme, entre la liberté des Anciens et la liberté des Modernes."

3. Voir cependant les conclusions très nuancées sur le sujet de M.-F. Bich, "Défense et illustration du droit québécois", Sociologie et sociétés, Montréal, vol. XXVI, no 2, automne 1994, p. 57-89.

4. R. Nozick, Anarchie, État et utopie, traduction d'É. d'Auzac de Lamartine, Paris, PUF, 1988, p. 363. 
les nuances que l'on devrait y apporter, une citation résume de façon incisive cette nouvelle donne:

Aucun gouvernement, même au Nord, n'exerce plus de contrôle sur les entreprises multinationales. Si une loi dérange leur expansion, elles menacent de partir, et elles peuvent le faire sur-le-champ. Elles sont libres de courir la planète pour choisir la main-d'œuvre la moins chère, l'environnement le moins protégé par la loi, le régime fiscal le moins onéreux, les subsides les plus généreux. Plus besoin de s'identifier à une nation ou de laisser un attachement sentimental (fut-il patriotique) entraver leurs projets. Elles se trouvent totalement hors de contrôle ${ }^{5}$.

Après l'effondrement du marxisme et au-delà du triomphe idéologique du libéralisme, tant politique qu'économique, nous entrevoyons, face à ces pathologies contemporaines, l'importance de réévaluer et de repenser la tradition républicaine soucieuse du foyer de l'unité identitaire de la société alors que la tradition libérale a largement escamoté ce problème, le prenant très superficiellement pour acquis ${ }^{6}$. Toutefois, tel n'est pas le but que je caresse ici, ni même celui d'examiner dans le détail les deux phénomènes plus récents qui fractionnent impitoyablement les allégeances communautaires et rendent plus ténues les chances de délibérer en commun sur les finalités et les valeurs auxquelles les communautés doivent adhérer. Je veux plutôt interroger en amont du libéralisme contemporain le lien intime qui unit le juridique et l'économique, mais, plus fondamentalement encore, saisir à sa source même les rôles de la fiction instituante de toute notre modernité politique, expliciter le sens du fiat inhérent à son projet de nous "fabriquer" en tant que sujet individuel autonome, lors même que ce projet prétend du même geste repousser à jamais les fictions et les mythes anciens, tant religieux que philosophiques, au profit

5. E. Goldsmith, "Une seconde jeunesse pour les comptoirs coloniaux. Quand les firmes transnationales imposent leur loi", Le Monde diplomatique, Paris, avril 1996.

6. Voir C. Taylor, Hegel et la société moderne, traduction de P. R. Desrosiers, Québec et Paris, les Presses de l'Université Laval et Cerf, 1998, p. 124, et F. Dumont, Raisons communes, Montréal, Boréal, 1995, chapitres 3, 4 et 5. Même un auteur contemporain comme J. Rawls, qui cherche à renouveler les philosophies du contrat social, prend pour prémisse de sa théorie de la justice une société moderne déjà constituée, ayant des frontières, des intuitions de mœurs libérales, etc. Le débat actuel entre les communautariens et les libéraux réactive de façon intéressante toute cette question, de même que les importants travaux de J. G. A. Pocock, Q. Skinner, etc. 
d'une "science politique". Après un survol des antécédents nécessaires à la compréhension de l'avènement de l'individu moderne chez Hobbes, j'examinerai chez lui et chez Locke le sens et la portée de la fiction instituante afin d'analyser et de légitimer le sens et le consentement au contrat social. Par où l'on verra, je l'espère, la pertinence de cette anamnèse afin d'y déceler les a priori les plus puissants de notre idéologie et sans doute aussi les ferments de notre échec canadien à fonder une réelle communauté politique. Peut-être, enfin, cet exercice sera-t-il salutaire en ce qu'il pemettra de nous dégriser quelque peu de certaines illusions modernes, nouvelles ombres auxquelles on s'identifie.

\section{Du mythe à la mise en scène du sacré}

Nous savons que les sociétés traditionnelles orales s'ordonnaient grâce à (et autour d')une fiction originaire, d'un mythe qui situe dans un passé reculé, en son ancienneté intangible, un audelà sacré où se donne la Loi. Le mythe, en effet, vient littéralement ordonner et structurer le monde et fournir une histoire sacrée, "réelle", où les exploits des dieux et des héros civilisateurs servent de modèles pour le comportement et l'organisation des communautés. Davantage, le mythe offre à la communauté ce qui est constitutif de son être commun, ce munus, ce "présent que l'on partage" comme l'indique l'étymologie. Des travaux contemporains ${ }^{7}$ ont pu ébranler la thèse largement reçue selon laquelle la modernité politique, la volonté de fonder le pouvoir sur un ordre autonome, choisi par les hommes eux-mêmes, avait été le fruit d'une rupture brutale contre le christianisme, contre l'hétéronomie du pouvoir divin et des règles théologiques. Certes, la critique s'est développée à l'intérieur du christianisme, témoin les schismes, mais c'est bien précisément parce que la possibilité même de cette critique gisait déjà au cour du christianisme: c'est "la religion de la sortie de la religion", celle qui rendra possible l'athéisme et qui, d'un point de vue politique, permettra le passage d'un ordre hétéronome, intégralement subi, vers un ordre autonome, de plus en plus voulu.

Le christianisme, en effet, brise l'ancienne dépendance envers un passé mythique en offrant un Dieu créateur qui vient s'incarner

7. Au premier chef M. Gauchet, Le désenchantement du monde. Une histoire politique de la religion, Paris, Gallimard, 1985. 
32

dans l'histoire, faisant ainsi surgir un présent et un salut à venir; mieux, cet événement ouvre l'histoire, un temps des hommes où la simple répétition du passé perd tout sens au profit des contours nouveaux du présent et de l'énigmatique futur que saint Paul veut aussitôt conjurer en supposant imminente la fin des temps. Mais une seconde rupture est introduite par le christianisme en ce que l'incarnation et la résurrection instaurent une séparation entre l'icibas et l'au-delà, entre le visible et l'invisible, là où il y avait auparavant continuité. Les hommes ne séjournent plus dans un monde enchanté où l'invisible vient sanctifier et justifier le visible, ils demeurent ici-bas au moment même où Dieu se retranche du monde, leur laissant la lourde tâche de comprendre pourquoi ils y vivent, pourquoi leur Dieu y tolère le mal, l'injustice, etc. Le sens n'est plus donné, il est à construire. Le désenchantement du monde signifie désormais l'interprétation du monde et son interrogation. Ce n'est pas à dire que le sens soit par ailleurs inaccessible. En effet, l'interrogation se détournera bientôt du monde extérieur au profit du for intérieur, du soi, là où s'éprouve, par la foi, la présence de l'absent, Dieu. La recherche de Dieu et de soi deviennent en effet une même épreuve, un travail de soi sur soi, une askésis, une ascèse ${ }^{8}$. Plusieurs écoles philosophiques grecques avaient insisté sur le souci de soi, mais ce soi n'avait pas encore les profondeurs de l'intériorité 9 . L'homme judéo-chrétien étant à la fois souffle spirituel et chair pécheresse, image de Dieu et être déchu suite à la tentation du Diable, l'introspection gagne dorénavant tout son sens en tant qu'examen de la présence divine en soi et volonté de débusquer les tentations en soi. Comme le résume Jean-Pierre Vernant :

Une nouvelle forme de l'identité prend corps à ce moment: elle définit l'individu humain par ses pensées les plus intimes, ses imaginations secrètes, ses rêves nocturnes, ses pulsions pleines de péché, la présence constante, obsédante, dans son for intérieur, de toutes les formes de tentation ${ }^{10}$.

8. Peter Brown est peut-être celui qui a le mieux éclairé la mise en place des conditions d'apparition du "soi intérieur" aux IV ${ }^{\mathrm{e}}$ et $\mathrm{v}^{\mathrm{e}}$ siècles après J.-C. Voir, de cet auteur, The Making of Late Antiquity et Society and the Holy in Late Antiquity, Londres, Cambridge University Press, 1978 et 1982.

9. Voir "P. Hadot: histoire du souci", Magazine littéraire, Paris, $\mathrm{n}^{\circ} 345$, juilletaoût 1996, p. 18-23.

10. L'individu, l'amour, la mort. Soi-même et l'autre en Grèce ancienne, Paris, Gallimard, 1996, p. 231. 
Il ne faudrait pas ignorer toutefois le contexte politique dans lequel se diffuse la philosophie stoïcienne à qui les chrétiens emprunteront, tout en les transposant, les idées de Cosmopolis et de Loi de la Nature ${ }^{11}$. Les conquêtes d'Alexandre marquent en effet l'éclatement de la polis, puis bientôt, en passant par les grandes monarchies hellénistiques, l'avènement de l'empire romain universel qui semblera donner, par le biais d'institutions, une armature juridique et administrative au vieux rêve des sages de la Cosmopolis. Pourtant, à défaut de l'antique citoyenneté, là où la liberté se vivait en tant que membre actif d'une communauté concrète, limitée mais autonome, l'individu de la civilisation romaine, réduit souvent à la passivité, réclame de nouvelles raisons de vivre soit à de nouvelles religions, soit à de nouvelles philosophies. De là, peut-être par compensation imaginaire, l'idée d'une loi naturelle divine, éternelle et immuable - d'où procéderaient les sociétés politiques particulières et leurs lois positives -, qui gouverne la Cité universelle idéale. La Cité idéale est une utopie qui sert d'abord à mesurer l'irréalité des autres cités qui rêvent du pouvoir, autant de fictions déformées et déformantes du véritable bonheur ${ }^{12}$. Chaque individu peut se considérer comme une bouture, comme un logos particulier du Logos universel dans le stoïcisme, comme uni à ces frères en une relation filiale à Dieu dans le christianisme.

Un dualisme, dès lors, est posé: une tension s'instaure entre l'idéal, le fictif pour nous si l'on veut, et le réel, entre le céleste et le terrestre, le surnaturel et le naturel, l'âme et le corps, la Cité de Dieu et les cités humaines, bref, entre autant d'oppositions constitutives du christianisme et de notre histoire où les deux pôles sont fortement hiérarchisés et ordonnés. Lorsque le Christ enseigne, en effet, de rendre à César ce qui est à César et à Dieu ce qui est à Dieu, la symétrie ne saurait qu'être apparente puisque l'ordre mondain est dévalué et relativisé; on ne se plie devant César que par respect pour Dieu qui institue toute autorité. C'est ici que se marque l'originalité de la situation occidentale; une Église va s'établir afin de guider les croyants dans leur quête

11. "Il ne peut y avoir ni Juif ni Grec [...] ni esclave ni homme libre [...] ni mâle ni femelle, car vous êtes tous un homme en Jésus-Christ" dit saint Paul. Voir notamment, sur cette question, Louis Dumont, Essais sur l'individualisme. Une perspective anthropologique sur l'idéologie moderne, Paris, Seuil, 1985, chapitre 1.

12. L'influence de Platon est évidemment patente ici. 
34

personnelle de Dieu, afin d'établir une interprétation adéquate du message et du sens divin, mais elle ne se bornera pas longtemps à ce rôle théologique et débordera rapidement dans le domaine de César. Ce problème théologico-politique est connu ${ }^{13}$. L'Église pose à la fois un problème conjoncturel et structurel. Le premier tient à ce que, devant l'effondrement et la désorganisation de l'empire face aux invasions barbares, l'Église a pris sur elle des fonctions sociales et politiques que les autorités légitimes ne remplissaient plus adéquatement. Le second est plus profond et s'étend sur plusieurs siècles. L'Église, comme "corps mystique du Christ", en assurant la médiation entre l'ordre divin et l'ordre humain, crée une situation de concurrence avec le monde de César. $\mathrm{Si}$, en effet, elle laisse libre ce domaine en ce qu'il ne l'intéresse pas, du moins pas prioritairement - le salut qu'elle apporte n'est pas de ce monde - , elle reste toutefois chargée par Dieu même de conduire les hommes au salut. Or, puisque toutes les actions humaines s'inscrivent forcément dans l'alternative du bien et du mal, l'Église a tendanciellement un droit de regard sur celles-ci; aussi réclamera-t-elle la plenitudo potestasis. Si cette rivalité entre les deux ordres aboutit tantôt à une coexistence harmonieuse, tantôt à des guerres qui useront l'Europe, elle laisse cependant ouvert, à la différence du judaïsme et de l'Islam, un espace possible d'affranchissemnt pour le domaine où se règlent les affaires terrestres: la politique et le droit pourront devenir des activités pleinement profanes et autonomes.

Cela étant, il demeure malgré tout un lieu par où le roi ou les autorités restent liés au divin, c'est celui du fondement même du pouvoir. Le monarque est roi de "droit divin", sa personne sacrée incarne l'invisible dans le visible. Même si ce rôle de liaison est au fond toujours déjà miné par l'incarnation du Christ - le roi ne saurait être au mieux que l'image du Christ —, il reste que le roi représente, à défaut du corps mystique, la nécessaire union particulière des sujets et met littéralement en scène le corps politique.

13. L. Strauss et son école ont particulièrement contribué par leurs travaux à redonner une place centrale à ce problème afin de comprendre l'histoire de notre modernité. Je m'inspire ici du premier chapitre du livre de P. Manent, Histoire intellectuelle du libéralisme. Dix leçons, Paris, Hachette, coll. "Pluriel ", 1997. 


\section{Théâtralité du politique : l'État fictif de Hobbes}

On mesurera sans doute mieux, suite à ces considérations, l'originalité de l'architecte des états modernes, Thomas Hobbes. Il se considéra comme le fondateur de la science politique, tenant pour une rêverie la longue et persistante tradition inaugurée par Socrate: "tout ce que les philosophes moraux ont écrit jusqu'à présent de la science n'a servi de rien", "ni Platon, ni, jusqu'ici, aucun autre philosophe, n'ont mis en ordre, et prouvé de façon adéquate ou seulement probable tous les théorèmes de morale ${ }^{14}$. Quoi qu'il en soit de cette prétention, il est certain que Hobbes opère une révolution profonde car là où Luther se contentait de remettre en cause, au nom de l'individu, le rôle de médiation de l'Église entre le chrétien et Dieu, notre philosophe va jusqu'à établir que le pouvoir n'est plus d'origine divine mais prosaïquement terrestre. Ce qui fonde la société n'est plus qu'un "contrat social" passé librement entre des individus égaux; le lien social y est conçu comme artificiel, volontaire et de nature essentiellement juridique. Le principe de constitution du corps politique ne reçoit plus sa légitimité d'en haut, il n'est plus donné, il n'emprunte plus la voie de la subordination hiérarchique des êtres, il est créé artificiellement d'en bas, il repose sur la souveraineté du peuple. Il naît et ne peut naître que d'un contrat entre des êtres naturels, primitivement déliés les uns des autres, indépendants; l'État "est comme une création tirée du néant par l'esprit humain ${ }^{15}$ ".

Cet extraordinaire travail de sape et de reconstruction scientifique de l'État se fait pourtant, chez Hobbes, grâce à un discours qui se donne explicitement - il ne s'agit donc pas d'un mythe sur le mode du comme si, sur le mode de la fiction rationnelle. La notion de fiction devient décisive dans la constitution du discours de la théorie politique moderne, de Hobbes à Rawls, en ce qu'elle permet une narration explicative des fondements

14. Respectivement De Cive [1642], épître dédicatoire, traduction de S. Sorbière, Paris, GF-Flammarion, p. 84, et Léviathan [1651], XXXI, traduction de F. Tricaud, Paris, Sirey, 1971, p. 392. Voir également Elements of Law, pré-

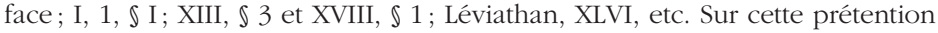
de Hobbes, voir le commentaire hostile de L. Strauss, Droit naturel et histoire, Paris, Flammarion, 1986, p. 153 et suivantes.

15. Elements of Law, II, 1, 1., ma traduction. J'utilise l'édition de J. C. A. Gaskin, Oxford, Oxford University Press, 1994. 
politiques qui rompt délibérément avec les anciens discours théoriques ${ }^{16}$. Les idées d'état, de nature, d'individu, de contrat social doivent effectivement leur apparition au registre discursif de la fiction car celui-ci permet de couper court aux idées d'hérédité, de tradition, de vertu, etc., autant de critères désormais congédiés pour penser la légitimité. Ce discours fictif ne saurait donc être assimilé avec le genre utopique car il ne prétend pas offrir un modèle d'État qui permettrait de corriger ou même de rejeter la pauvre réalité; il ne saurait non plus être assimilé aux miroirs des princes car il n'exhorte pas les gouvernants à se conformer à un idéal. Hobbes prolonge et radicalise Machiavel qui entendait "suivre la vérité effective des choses " plutôt que se perdre à imaginer des républiques fictives ${ }^{17}$. La fiction rationnelle qu'il met en œuvre se modèle en effet sur la mise à distance que la science moderne opère à l'égard du monde physique réel et qualitatif. Cette annihilatio mundi permet de penser des êtres abstraits (l'homme naturel, le droit, etc.) afin de donner accès à la structure juridique de l'État (ses limites, la répartition des droits et des devoirs) et à son institution qui ne saurait faire l'économie de la narration, du roman de formation ${ }^{18}$. Elle fournit donc la clé du fonctionnement politique, celui d'une obéissance incontestable qui assurera la paix civile, c'est-à-dire la conservation de soi.

Dès la préface du De Cive, Hobbes nous donne à voir d'un même geste et sa méthode analytique ou, pour prendre les termes de l'époque, la méthode résolutive-compositive, et l'acte de naissance de l'individu moderne. Afin d'atteindre la "première origine de la justice", Hobbes va soumettre l'État à une analyse comparable au démontage d'une horloge en chacune de ses parties:

ainsi en la recherche du droit de l'État, et du devoir des sujets, bien qu'il ne faille pas rompre la société civile, il la faut pour-

16. J.-J. Rousseau, Discours sur l'origine et les fondements de l'inégalité parmi les hommes, dans Euvres complètes, Paris, Gallimard, vol. III, p. 132-133: "Commençons donc par écarter tous les faits, car ils ne touchent point à la question. Il ne faut pas prendre les Recherches, dans lesquelles on peut entrer sur ce sujet pour des vérités historiques, mais seulement pour des raisonnements [...] plus propres à éclaircir la Nature des choses qu'à montrer leur véritable origine." Voir Y. C. Zarka, Philosophie et politique à l'âge classique, Paris, PUF, 1998, p. 225.

17. Le Prince, XV, Euvres, édition de C. Bec, Paris, Laffont, 1996, p. 148.

18. F. Tricaud, "Le roman philosophique de l'humanité chez Hobbes et chez Locke", Archives de philosophie, Paris, n 55, 1992, p. 631. 
tant considérer comme si elle était dissoute, c'est-à-dire qu'il faut bien entendre quel est le naturel des hommes, qu'est-ce qui les rend propres ou incapables de former des cités, et comment c'est que doivent être disposés ceux qui veulent s'assembler en un corps politique ${ }^{19}$.

On le voit, l'origine de l'État, c'est l'homme sans l'État, c'est l'état naturel de l'homme, c'est la nature humaine ${ }^{20}$. L'individu moderne naît en 1642 de la décomposition abstraite du corps social; il existe dès lors avant toute inscription dans un corps sexué, une tradition ou une hiérarchie sociale, avant toute obéissance à une loi politique ou religieuse. Tel est abstraitement l'homme moderne, tel est l'homme contemporain en sa conscience: il est homme avant d'être citoyen et avant d'être chrétien. Mais ne brûlons pas les étapes et revenons à la conception artificialiste de l'État, c'est-à-dire à la reconstruction théorique de l'État une fois analysées ses différentes parties, une fois atteinte, par le recours au comme si, par le recours à la fiction rationnelle, la "nature" des choses ${ }^{21}$. C'est dans la fameuse introduction du Léviathan que la conception artificialiste de l'État est proclamée avec le plus de force de manière synoptique :

La nature, cet art par lequel Dieu a produit le monde et le gouverne, est imitée par l'art de l'homme en ceci comme en beaucoup d'autres choses, qu'un tel art peut produire un animal artificiel. [...] Mais l'art va plus loin, en imitant cet ouvrage raisonnable et le plus excellent, de la nature: l'homme. Car c'est l'art qui crée ce grand Léviathan qu'on appelle République ou État, lequel n'est qu'un homme artificiel, quoique d'une stature et d'une force plus grandes que celles de l'homme naturel, pour la défense et protection duquel il a été conçu; en lui la souveraineté est une âme artificielle, puisqu'elle donne vie et mouvement à l'ensemble du corps; les magistrats et les autres fonctionnaires préposés aux tâches judiciaires et exécutives sont les articulations artificielles; la récompense ou le châtiment qui,

19. Op. cit., p. 71.

20. Le père de la psychanalyse, Freud, retiendra la leçon dans Malaise dans la civilisation où souffle partout l'esprit de Hobbes, de la représentation des liens entre l'indivdu et la société jusqu'à la conviction atomistique d'un individu présocial: "La liberté individuelle n'est donc nullement un produit culturel. C'est avant toute civilisation qu'elle était la plus grande...". trad. C. et J. Odier, Paris, PUF, 1983, p. 45. C'est dire l'influence de la fiction hobbesienne sur les sciences humaines...

21. Voir, par exemple, Elements of Law, II, III, 2. 
attachées au siège de la souveraineté, meuvent chaque articulation et chaque membre en vue de l'accomplissement de sa tâche, sont les nerfs, car ceux-ci jouent le même rôle dans le corps naturel; la prospérité et la richesse de tous les membres particuliers sont la force; la sauvegarde du peuple est son occupation, les conseillers qui proposent à son attention toutes les choses qu'il lui faut connaître, sont sa mémoire; l'équité et les lois lui sont une raison et une volonté artificielles; la concorde est sa santé, les troubles civils sa maladie, et la guerre civile sa mort. Enfin les pactes et conventions par lesquels les parties de ce corps politique ont été à l'origine produites, assemblées et unifiées ressemblent au Fiat ou Faisons l'homme que prononça Dieu lors de la création ${ }^{22}$.

À l'évidence, un long commentaire s'imposerait, mais je me restreindrai à l'essentiel pour mon propos. Si la dernière phrase renvoie évidemment à la Genèse, il faut aussi y saisir l'étymologie du mot fiction qui fait résonner toute la conception mécaniste de Hobbes dans ce contexte, fictus signifiant "modeler dans l'argile, reproduire, inventer". Cet automate, cet État, ce "dieu mortel", imite un corps qui est déjà lui-même le fruit d'une construction artificielle pour l'esprit qui essaie d'en percer les secrets de fonctionnement. "L'homme, poursuit-il aussitôt, est matière et artisan [artificer] de lui-même". Existe-il meilleure définition de la souveraineté? De plus, il faut saisir l'imbrication étroite qui lie ici le mécanisme et le nominalisme médiéval tardif. En effet, l'irruption, en théologie, du volontarisme permet de se libérer progressivement de la conception du monde en tant que "cosmos" hiérarchisé, pénétré de raison et au sein duquel les valeurs objectives s'imposeraient à la volonté de Dieu lui-même. La souveraineté de Dieu, croit-on, serait incompatible avec pareil (ancien) ordre naturel intrinsèque qui déterminerait par lui-même le bien et le mal; il lui appartient de déterminer souverainement ce qui est bien. La conception hobbesienne de la liberté semble transférer cette prérogative divine à l'homme qui s'assigne à lui-même ses propres fins sans intervention d'une autorité extérieure. Le droit politique se fait désormais par décret souverain, par un fiat prométhéen. Par où l'on voit que cet artificialisme volontaire est le doublet d'une puissante affirmation technicienne qui étend jusqu'à la politique le célébrissime mot d'ordre cartésien: "nous serons comme mâ̂tres et possesseurs de la nature".

22. Léviathan, p. 5-6. 
On a maintes fois souligné les liens entre le courant nominaliste et l'essor du mécanisme dont Hobbes est entièrement pénétré et un des principaux artisans. Mais il y a plus, car la représentation moderne d'une nature désenchantée et mécanisée favorise également la pensée d'un individu sans appartenances puisqu'il n'y a plus aucune finalité objective susceptible de lui assigner, comme jadis, la place qui lui revient dans la hiérarchie sociale, image de la hiérarchie naturelle. Il ne saurait donc y avoir davantage de bien en soi :

car il n'existe rien qui soit tel, simplement et absolument; ni aucune règle commune du bon et du mauvais qui puisse être empruntée à la nature des objets eux-mêmes: cette règle vient de la personne de chacun, là où il n'existe pas de République, et, dans une République, de la personne qui représente celle-ci ${ }^{23}$.

L'homme, désormais, n'a plus à chercher l'ordre au sein des choses, il ne peut être que le produit du travail de l'esprit humain; le bien n'est plus une norme d'excellence indépendante de notre volonté puisque, très précisément, la volonté de chacun détermine son bien.

C'est en fonction de ces données qu'il convient d'interpréter la fiction rationnelle qu'offre Hobbes de l'état de nature dans lequel le pacte social est conclu. Dans un tel état, il n'y a pas de place pour une "activité industrieuse" ou pour un "usage des richesses", pas de "constructions commodes", "pas d'arts; pas de lettres; pas de société; et ce qui est le pire de tout la crainte et le risque continuels d'une mort violente; la vie de l'homme est alors solitaire, besogneuse, pénible, quasi animale, et brève ${ }^{24}$ ". Temps sans loi donc, temps sans lien social, temps barbare. C'est parce qu'il est intolérable que les hommes décident de le quitter en se soumettant volontairement à la souveraineté de Léviathan. Trois raisons expliquent, selon Hobbes, cet état misérable: 1. la lutte incessante pour le pouvoir, 2. l'égalité qui règne entre les individus, et 3. l'absolue liberté dont ils jouissent.

1. "Ainsi, je mets au premier rang, à titre d'inclination générale de toute l'humanité, un désir perpétuel et sans trêve d'acquérir pouvoir après pouvoir, désir qui ne cesse qu'à la mort ${ }^{25}$ ". Contre l'héritage païen, Hobbes estime que:

23. Ibid., I, 6, p. 48.

24. Op. cit., p. 125.

25. Ibid., p. 95. 
la félicité de cette vie ne consiste pas dans le repos d'un esprit satisfait. Car n'existent en réalité ni ce but dernier ni ce bien suprême dont il est question dans les ouvrages des anciens moralistes. [...] La félicité est une continuelle marche en avant du désir, d'un objet à un autre, la saisie du premier n'étant encore que la route qui mène au second. La cause en est que l'objet du désir de l'homme n'est pas de jouir une seule fois et pendant un seul instant, mais de rendre à jamais sûre la route de son désir futur. [...] La compétition dans la poursuite des richesses, des honneurs, des commandements et des autres pouvoirs incline à la rivalité, à l'hostilité et à la guerre, parce que le moyen pour un compétiteur d'atteindre ce qu'il désire est de tuer, d'assujettir, d'évincer ou de repousser l'autre ${ }^{26}$.

On le voit, la moralité humaine, le sens du bien et du mal, n'a plus de support dans la nature de l'homme. La félicité n'a pas et ne peut avoir de définition universelle, car elle renvoie aux désirs propres à chaque homme, infiniment variables, qui ne connaissent de fin qu'avec la mort de celui qui les éprouve. "Là où il n'est pas de loi, affirme-t-il dans un passage célèbre, il n'est pas de justice ${ }^{27}$ ". Hobbes rompt ainsi avec tout l'héritage ancien et chrétien du droit naturel.

2. La lutte et la compétition sont d'autant plus âpres qu'il existe une "égalité des aptitudes" entre les individus, sinon la guerre de tous contre tous s'achèverait par la victoire des plus forts et l'asservissement des plus faibles. Cela n'est pourtant pas le cas puisque souvent les rusés triomphent des plus forts. Constatant cette "égalité dans l'espoir d'atteindre [ses] fins ${ }^{28}$ ", chacun est conduit à craindre les autres et à chercher à s'en protéger par l'accroissement de son pouvoir. De l'égalité procède la défiance et de la défiance la guerre: chaque homme a intérêt à "se rendre maître, par la violence ou par la ruse, de la personne de tous les hommes pour lesquels cela est possible ${ }^{29}$ ". Voilà ce qu'exige la conservation de soi. Plusieurs considèrent ici l'exposé de Hobbes plus ou moins convaincant, mais il ne faudrait pas en rester ainsi à la surface de l'exposé car l'enjeu essentiel est ailleurs; il s'agit de rompre avec la hiérarchie "naturelle" des hommes au profit de l'égalité "naturelle" de tous

26. Ibid., p. 95-96.

27. Ibid., p. 126.

28. Ibid., p. 122.

29. Ibid. 
dans le consentement mutuel du pacte social. Hobbes fonde l'idée démocratique de l'acte instituant parce qu'il fonde la notion de souveraineté sur le consentement des individus égaux entre eux. La fiction rationnelle permet de faire tabula rasa de toutes les hiérarchies anciennes.

L'inégalité présente a été introduite par les lois civiles. Je sais bien qu'Aristote, au premier livre de sa Politique, pose comme fondement de sa doctrine que les hommes sont par nature, les uns plus dignes de commander, les autres d'être commandés: comme si le maître et le serviteur ne tiraient pas leur origine du consentement des hommes, mais bien d'une différence d'esprit; ce qui n'est pas seulement contraire à la raison, mais contraire aussi à l'expérience. [...] Si donc la nature a fait les hommes égaux, cette égalité doit être reconnue; et si elle les a faits inégaux, étant donné que les hommes, se jugeant égaux, refuseront de conclure la paix, si ce n'est sur un pied d'égalité, cette égalité doit néanmoins être admise ${ }^{30}$.

3. Enfin, l'état de nature est intolérable parce que chacun jouit d'une liberté sans limites de poursuivre ses propres fins. Il y règne le droit de nature: "la liberté qu'a chacun d'user comme il le veut de son propre pouvoir, pour la préservation de sa propre nature [...] et de faire tout ce qu'il considérera, selon son jugement et sa raison propres, comme le moyen le mieux adapté à cette fin ${ }^{31}$ ". Le passage nécessaire à l'état civil est donc tracé; il s'agira non pas de renoncer à toute liberté, mais de restreindre commutativement cette liberté naturelle illimitée autant qu'il sera exigé par Léviathan afin d'assurer la coexistence pacifique des atomes individuels $^{32}$. Là où la loi n'exige rien, là où la loi demeure silencieuse, l'individu reste libre ${ }^{33}$. Hobbes fonde donc la conception négative, la conception libérale de la loi comme artifice extérieur aux individus. Il ne s'agit pas de renoncer à poursuivre les fins que les hommes ont dans l'état de nature. C'est au contraire afin de les rendre plus aisément accessibles et afin d'en assurer la jouissance que les individus se soumettront à Léviathan qui décrètera lesquelles seront injustes.

\section{Ibid., p. 154.}

31. Ibid., p. 128.

32. Ibid., p. 129: "Que l'on consente, quand les autres y consentent aussi, à se dessaisir, dans toute la mesure où l'on pensera que cela est nécessaire à la paix et à sa propre défense, du droit que l'on a sur toute chose."

33. Ibid., chapitre XXXI. 
Nous en arrivons donc à cet autre moment essentiel du roman de formation hobbesien, celui du contrat social. Ce pacte ne prétend évidemment pas être un événement historique. Il s'agit bien plutôt, encore une fois, d'une reconstruction qui vise à fournir l'extension possible de la légitimité, à répartir les droits de Léviathan et les devoirs des sujets que nous n'avons pas à examiner ici. La seule façon, selon Hobbes, d'ériger un pouvoir commun "est de confier tout le pouvoir et toute la force à un seul homme ou à une seule assemblée, qui puisse réduire toutes les volontés [...] en une seule volonté ${ }^{34}$ ". Ce contrat, qui réalise l'unité, est formulé significativement selon le registre discursif du comme si:

c'est comme si [as if] chacun disait à chacun: j'autorise cet homme ou cette assemblée, et je lui abandonne mon droit de me gouverner moi-même, à cette condition que tu lui abandonnes ton droit et que tu autorises toutes ses actions de la même manière. Cela fait, la multitude ainsi unie en une seule personne est appelée une RÉPUBLIQUE ${ }^{35}$.

C'est à partir de cette convention, de cette scène primitive de l'autorisation, que Hobbes fait surgir une nouvelle couche de sens à la fiction pour fonder la théorie moderne de la délégation du pouvoir, de la représentation, et celle de l'unité de l'État. En effet, dans le chapitre XVI, Des personnes, des auteurs et des êtres personnifiés, après avoir recensé les diverses acceptions de personne dans les langues anciennes, son évolution du théâtre au tribunal, etc., il distingue entre l'auteur et l'acteur: lorsque l'acteur parle et agit en son nom propre, il est une personne naturelle; lorsque l'acteur parle et agit au nom d'un autre, "on parle d'une personne fictive ou artificielle ${ }^{36}$ ". Or le souverain doit personnifier le pouvoir délégué par la multitude en devenant cette personne fictive qui fait passer les individus de l'état de nature à l'unité de l'état civil, celui qui parle en leur nom. Les individus, autant d'auteurs d'une impossible pièce cacophonique dans l'état de nature, doivent être représentés par un acteur autorisé qui met en scène et assume l'unité de la parole qui dicte la loi :

car c'est l'unité de celui qui représente, non l'unité du représenté, qui rend une la personne. Et c'est celui qui représente qui assume la personnalité, et il n'en assume qu'une seule. On

34. Ibid., p. 177.

35. Ibid.

36. Ibid., p. 161. 
ne saurait concevoir l'unité dans une multitude sous une autre forme ${ }^{37}$.

On comprend mieux, dès lors, pourquoi Hobbes parle de l'État comme d'un homme artificiel ou fictif. Conformément aux deux sens de to act, c'est la personne fictive qui joue, qui théâtralise la multitude, et qui agit en son nom. Nul doute que Hobbes y songeait en proclamant son fiat inaugural. Mais, à la réflexion, le souverain fait davantage qu'agir au nom des auteurs dans la mesure où il devient par là même l'auteur de la loi. Il incarne l'unité juridique. À défaut de tout ancrage du bien et du mal dans une nature ordonnée, il revient à la personne fictive de fabriquer une loi comme mécanisme artificiel de régulation des atomes. Nous saisissons ici à sa source la conception libérale de la loi comme artifice extérieur aux individus. Mais, par là même, Hobbes inverse le rapport initial auteur/acteur car le souverain devient véritablement le seul auteur à dire le droit, à autoriser les lois ${ }^{38}$. Les auteurs initiaux deviennent ainsi sujets aux obligations et aux commandements du souverain. La délégation du pouvoir est à ce prix, mais il reste entendu que si le souverain ne peut assurer la sécurité des sujets, prémisse du contrat social, ceux-ci peuvent recouvrer leur droit naturel illimité. Mieux, les sujets ont toujours ce droit dans le silence de la loi.

Mais une dernière conséquence du contrat social mérite d'être soulevée brièvement. Car si le souverain est le seul auteur de la loi, comment faut-il entendre son rapport à Dieu?

Le problème que pose l'exégèse de la Bible occupe la moitié du Léviathan parce que Hobbes doit réinterpréter tout l'héritage chrétien de telle sorte que la loi divine révélée se confonde avec l'obéissance au Souverain. "Tout ce qui est nécessaire au salut est contenu dans deux vertus: la foi dans le Christ et l'obéissance aux lois ${ }^{39}$ ". Comment cela? La foi dans le Christ se ramène simplement à deux choses, affirme Hobbes à la fin d'une étourdissante érudition exégétique: l'espérance de l'avènement de son Royaume où il sera roi et la règle d'or de la réciprocité ("Ne fais pas à autrui ce que tu ne voudras pas qu'on te fît"). L'obéissance

37. Ibid., p. 166.

38. Ibid., p. 291. Voir Y. C. Zarka, Hobbes et la pensée politique moderne, Paris, PUF, 1995, p. 208 et suivantes.

39. Ibid., p. 606. 
44

aux lois fut problématique pendant des siècles car les chrétiens voyaient double: fallait-il obéir au Souverain ou au Pape? À cette lancinante question, Hobbes répond que seul le Souverain est autorisé car le Christ n'a jamais revendiqué de pouvoir sur les hommes, son Royaume n'étant pas de ce monde. L'Église ne saurait donc réclamer pareil pouvoir, elle doit se borner à enseigner le salut. Il y aura donc autant d'églises que de Républiques. Hobbes entend ainsi soumettre définitivement le pouvoir ecclésiastique au pouvoir temporel et il entend couper court aux interminables guerres de religion qui rendaient impossible la paix civile.

Hobbes, donc, "invente" l'individu et l'état modernes parce qu'il attaque et rompt consciemment avec tout l'héritage politique classique et chrétien. Le citoyen grec est de ce monde, d'une communauté particulière, mais, en vertu de la hiérarchie sociale qui croit redoubler la hiérarchie naturelle, il est inégalitaire. Le chrétien n'est pas de ce monde, il n'est d'aucune patrie particulière, mais il est égalitaire dans le corps du Christ, dans le Royaume céleste. L'individu est ni l'un ni l'autre tout en étant par ailleurs l'un et l'autre; mieux, il est l'un contre l'autre ${ }^{40}$ : il est de ce monde et égalitaire, mais il n'est d'aucune patrie, il est le fruit de l'abstraction fictionnelle. L'individu hobbesien n'est plus le héros patriotique, ni le saint qui renonce à la gloire terrestre. Il deviendra bientôt le propriétaire et le consommateur fuyant tout sacrifice à l'une et à l'autre traditions. Mais, dès lors, la recherche de la cohésion sociale devient plus problématique car, si la société artificielle est fondée sur des individus indépendants, qu'estce qui lui permet de rappeler la dépendance de chacun à cette communauté-ci, ses devoirs sociaux et politiques lorsque tout pouvoir délégué se matérialisera progressivement sous la forme impersonnelle de la loi, cet artifice extérieur?

\section{L'individu moderne comme propriétaire et porteur de droits naturels}

À l'inverse de Hobbes, Locke postule l'aspect historique de l'état de nature afin d'analyser le fondement des droits individuels ainsi que l'extension et les limites du pouvoir politique. Il con-

40. Telle semble être l'intuition centrale du livre sans doute le plus personnel de P. Manent, La cité de l'homme, Paris, Fayard, 1994. 
teste et modifie le commencement et la fin de la construction hobbesienne: l'état de nature devient chez lui un état pacifique de parfaite égalité afin de doter l'individu des droits naturels "intrinsèques", et il justifie un droit de résistance au peuple lorsque le gourvernement dévie gravement de son mandat ( $\$ 243$ ) et trangresse la loi naturelle qui agit désormais comme une norme transcendante: "là où le droit finit, la tyrannie commence" $(\$ 202)^{41}$. Ces modifications, ainsi que les doctrines de la séparation des pouvoirs, de la propriété, etc., achèvent, selon l'historiographie classique, la mise en place de la synthèse libérale par Locke.

Quelques mots seulement sur cet état de nature seront nécessaires en guise d'introduction à notre propos, soit celui d'examiner la genèse du droit de propriété chez Locke, véritable acte de naissance de l'économie politique moderne - non seulement Smith, Malthus et Ricardo y trouveront leur source, mais tout le néolibéralisme s'y adosse ${ }^{42}$. Locke postule que cet état en est un d'égalité et de parfaite liberté: les hommes "sont parfaitement libres d'ordonner leurs actions, de disposer de leurs biens et de leurs personnes comme ils l'entendent, dans les limites de la loi naturelle, sans demander l'autorisation d'aucun autre homme ni de dépendre de sa volonté" ( $(6)$. "There being nothing more evident ${ }^{43}$." Cet état originaire, donc, "Soumis à la loi naturelle qui oblige chacun", sert de critère de justice avant tout pacte social. Si, chez Hobbes, il n'y a pas de justice avant la loi déterminée par Léviathan, ici, le pouvoir souverain doit se conformer à cette justice qui provient ultimement de Dieu. Là où le christianisme

41. Le second traité du gouvernement. Essai sur la véritable origine, l'étendue et la fin du gouvernement civil. Nous citons habituellement l'édition de J.-F. Spitz, Paris, PUF, 1994, mais nous faisons parfois des modifications à partir de l'édition critique des Two Treatises of Government [1690] qui fait désormais autorité, celle de P. Laslett, Cambridge University Press, 1965. Sur le droit de résistance, voir l'excellent article de N. Tarcov, "Locke's Second Treatise and the best fence against rebellion ", Review of Politics, Notre-Dame (Indiana, USA), vol. 43, avril 1981, p. 198-217.

42. Comme le résume Louis Dumont en traitant de la théorie lockéenne: "c'est sous l'aspect de la possession ou de la propriété que l'individualisme lève la tête, abat tout ce qui restait de la soumission de fait et de hiérarchie idéale dans la société, et s'installe lui-même sur le trône ainsi vidé". Homo aequalis. Genèse et épanouissement de l'idéologie économique, Paris, Gallimard, 1971, p. 75. Voir également l'ouvrage de C. B. Macpherson, La théorie politique de l'individualisme possessif de Hobbes à Locke, Paris, Gallimard, 1971.

43. "Rien n'est plus évident." Jefferson, au xviII siècle, et les rédacteurs des droits de l'homme, au $\mathrm{xx}^{\mathrm{e}}$ siècle, retiendront la leçon. 
46

enseignait l'égalité des hommes avant la Chute et pour le Royaume à venir, maintenant cette égalité et cette liberté sont des états naturels que les gouvernements doivent respecter. Davantage, ils n'existent que pour satisfaire ces droits. Nous vivons encore les effets de cette révolution dans l'histoire.

Que dit cette loi naturelle? "Chacun est tenu de se conserver soi-même [et] il doit, autant qu'il peut, préserver le reste du genre bumain." Cette conservation de soi et d'autrui nous oblige, car nous sommes "la propriété d'un seul Ouvrier tout-puisssant et infiniment sage" ( $(6)$. Mais par un acte de sa volonté, Dieu a donné la Terre aux fils des hommes. Sur une terre à l'origine indivise, où tout appartient à tous, il faut donc trouver le premier point, la légitime appartenance de l'homme, d'où le droit pourra se propager. Ce premier point, c'est la propriété que chaque individu a, par la loi naturelle, sur sa personne, sur son propre corps, qui implique celle de son travail, qui est usage du corps; donc des fruits du travail ${ }^{44}$. Le droit de propriété est essentiellement antérieur à l'institution de la société car il ne dépend pas du consentement d'autrui ou de la loi politique; le droit de propriété s'attache donc à l'individu seul, il est naturel. Ce point est fondamental pour Locke et pour tout le libéralisme économique car l'bomme est d'abord travailleur et propriétaire avant d'être un citoyen. Locke rompt avec la tradition, dans laquelle s'inscrit Hobbes, qui fait de la propriété un droit social, réglé et limité par les devoirs sociaux et politiques. Il est vrai que Locke semble d'abord admettre une limitation naturelle du droit naturel de propriété. Mais la suite de son exposé démontre que l'invention de la monnaie annihile, pour ainsi dire, les deux limites qu'il avait d'abord posées. Il vaut la peine de s'y attarder car cette genèse présentée comme historique nous semble plutôt constitutive d'une généalogie discursive, donc fictive, chargée de fonder le droit à l'appropriation illimitée ${ }^{45}$. Se délestant progressivement de

44. "Si la terre et toutes les créatures inférieures appartiennent à tous, du moins chaque homme détient-il un droit de propriété sur sa propre personne; et sur elle aucun autre que lui n'a de droit." "Le travail de son propre corps et l'œuvre de ses mains, peut-on dire, lui appartiennent en propre" ( $\$ 27$ ).

45. À partir de $\$ 100$, Locke examine longuement l'objection selon laquelle l'histoire n'offre aucun exemple de cet état de nature où les hommes indépendants et égaux auraient contracté entre eux afin d'instituer un gouvernement servant à protéger leurs propriétés. Il réplique qu'il y a toujours un gouvernement avant qu'il n'y ait des archives, car les lettres apparaissent 
tout l'ancrage religieux de ce récit, le libéralisme retiendra seulement le résultat. Mais comment croire à ce récit sans croire en Dieu? Du droit de l'Ouvrier divin à la sacralisation du droit du propriétaire, il n'y a qu'un pas, non? Il ne faut pas trop chipoter sur les fondements...

"À l'origine", la nature présente un état d'abondance avec ses vastes territoires vierges et une population peu nombreuse. Dans cet état, les pommes que vous cueillez sont vôtres car vous avez combiné ce qui n'appartient qu'à vous, le travail de votre cueillette, avec ce qui appartient à tous, les pommes pendant aux arbres ou gisant sur le sol. Dès lors, se pose l'objection suivante: n'est-ce pas priver les autres de ce bien? Non, car "les autres disposent, en quantité suffisante, de biens communs de même qualité" (\$27). Qui conteste votre propriété, conteste votre travail - et sur ce travail, il n'a aucun droit. "Car ce travail est la propriété indiscutable du travailleur, et personne d'autre que lui n'a le droit d'en récolter les fruits; du moins tant que les autres disposent, en quantité suffisante, de biens communs de même qualité" (\$27). Voilà donc la première limitation au droit de propriété : je dois en laisser aux autres de telle sorte qu'ils puissent s'approprier à leur tour les fruits de la terre en aussi grande quantité et en aussi grande qualité. Cette limitation découle du deuxième aspect de la loi de nature. D'autre part, je ne puis m'approprier plus que je puis consommer; ce serait gaspiller, or "Dieu n'a rien créé pour que l'homme le gaspille ou le détruise" ( $\mathbb{S} 31$ ). Voilà la deuxième limitation à l'appropriation. Le gaspillage semble d'abord une faute morale avant de se présenter comme un dommage économique ${ }^{46}$.

seulement après les arts plus nécessaires qui donnent la sécurité, l'aisance et l'abondance. C'est dire que la théorisation lockéenne de l'état de nature est une "reconstruction" où la loi naturelle, d'origine divine, est fondatrice. L'origine échappe toujours à la communauté, sa recherche suppose toujours la perte du souvenir, l'oubli. Cet aveu de Locke est aussitôt tempéré par l'affirmation suivante: "les documents relatifs à l'origine [...] illustrent clairement le mode de fondation que j'ai indiqué" ( $\$ 101)$. La démonstration qui suit semble aujourd'hui tout à fait fantaisiste, mais Locke écrit, presque furtivement, qu' "un argument qui conclut de ce qui a été à ce qui doit être est sans grande force" ( $\$$ 103). Locke est-il incohérent ou dissimulateur? Je penche en faveur de la première hypothèse: l'empririsme de Locke ne peut se réconcilier avec ses prémisses chrétiennes et son déductivisme en politique.

46. Contrairement à ce qu'affirme P. Manent, Histoire intellectuelle du libéralisme, p. 98, qui néglige ici curieusement la dimension éthico-religieuse chez Locke. Locke fait sienne la tradition biblique qui élève le travail au rang de 
La propriété de la terre, dans le domaine commun, est acquise de la même façon que les fruits de la terre. "L'étendue de terre qu'un homme laboure, plante, amende, cultive et dont il peut utiliser les produits mesure celle de sa propriété" (\$32). Cette appropriation est légitime car Dieu ne souhaite pas que la terre reste "commune et sans culture. Il l'a donnée pour l'usage de ceux qui seraient industrieux et rationnels, et non pour satisfaire le caprice et l'avidité [...] des querelleurs et chicaniers" (\$ 34). "La terre sans le travail ne vaudrait rien" ( $\$ 43)$. C'est la composante privée, le travail, qui donne de la valeur aux choses. Deux raisons font que les provisions naturelles ont peu de valeur:

1. une pomme n'est utile que cueillie. D'où une forme de paradoxe dans l'exposé de Locke: l'abondance qui caractérise la nature pendant "les premiers âges du monde" semble, vue de plus près, une pénurie.

Les Indiens qui sont riches en terres, mais pauvres de tous les conforts de la vie, la nature les a pourvus aussi généreusement que n'importe quel autre peuple des matières premières de l'abondance, c'est-à-dire d'un sol fertile et capable de produire avec libéralité ce dont on peut tirer nourriture, vêtement ou agrément; pourtant, faute de l'améliorer par le travail, ces nations ne possèdent pas la centième partie des commodités dont nous jouissons. Là-bas, le roi d'un territoire vaste et fertile est moins bien nourri, logé et vêtu qu'un journalier en Angleterre ( $\$ 41)$.

2. la très grande abondance fait en sorte que les choses sont sans grande valeur. Faute de moyens pour disposer de ses surplus agricoles avant qu'ils ne périssent, le travailleur n'accroîtra pas son bien. La limite de la juste propriété ne consiste donc pas dans l'excès de l'extension de la possession, mais dans son emploi manqué ou partiel.

Heureusement, l'invention de la monnaie permet de rendre incorruptible toute quantité de biens corruptibles. L'origine de cette convention est dans un désir naturel de l'individu: le désir de rendre incorruptibles les biens qu'il a produits au-delà de sa

commandement et repère dans la réussite positive des ouvres de l'homme la manifestation de la gloire et de la faveur divines, par l'adoption de la parabole des talents qui engage l'homme à agir pour le mieux en fonction du devoir qui lui a été confié. Cela étant, en pratique le résulat demeure le même... Voir S. Goyard-Fabre, "La propriété dans la philosophie de Locke", Archives de philosophie, Paris, n 55, 1992, p. 607-630. 
capacité de consommation pour les échanger contre d'autres biens de consommation qui lui font défaut. Elle est liée au désir de conservation - premier aspect de la loi de nature - , elle est un artifice pour satisfaire ce désir naturel plus complètement. C'est pourquoi Locke s'évertue à présenter l'invention de la monnaie comme le produit d'une progression "naturelle": les hommes échangent des biens périssables (des pommes) contre des biens plus durables (des noix); ils échangent ensuite des biens contre "une pièce de métal dont la couleur leur plaisait" (\$ 46); ils parvinrent à un accord que les choses rares, telles l'or et l'argent, seraient prises en échange pour des biens périssables ( $\$ 47)$.

Non seulement l'invention de la monnaie permit les échanges, mais les hommes, d'un commun consentement dont font état les textes bibliques (Locke se réfère explicitement à Genèse, 13, 5 et $36,6$. ), distinguèrent leurs territoires et, en vertu de lois et de conventions, commencèrent d'assigner des limites juridiques à leurs propriétés respectives $(\$ 38)$ en tenant compte de la valeur fiduciaire que le travail avait conférée à leurs arpents de terre. Seulement, très vite, le désir de posséder toujours plus développa en chacun la tendance à augmenter ses possessions. Alors, l'égalité primitive disparut et l'inégalité dans la possession privée des richesses s'installa. Bref, les terres inconnues deviennent rares parce que les terres clôturées s'accroissent, la production s'accroît et permet de supporter une population accrue $(\$ 48)$, la propriété s'agrandit (\$49) et l'égalité cède enfin la place à l'inégalité :

[...] par consentement mutuel, les hommes ont élaboré et adopté un procédé qui permet à chacun, légitimement et sans causer de tort, de posséder plus qu'il ne peut utiliser lui-même: pour le surplus, il reçoit de l'or et de l'argent, qu'on peut thésauriser sans nuire à personne [...]. Ce partage dans l'inégalité des possessions particulières, les hommes l'ont rendu possible en dehors des liens de la société, sans contrat (\$50).

Cette "reconstruction" de l'invention de la monnaie, en tant que relation contractuelle avant toute institution politique, n'est évidemment pas innocente, car Locke a fait davantage qu'expliquer l'origine de la propriété, il a justifié l'inégalité des possessions. Avec l'invention de la monnaie, l'homme "pouvait amasser autant qu'il le voulait de ces biens durables" ( $\$ 46)$. Celui qui, ne "produisant" pas, vit de l'achat et de la vente des biens est légitimement propriétaire: il ne vole personne, n'enlève rien à la quantité de valeur disponible dans la société, mais au contraire la 
conserve, la met en circulation et. pour autant, tend à l'accroître. "La croissance des terres et le bon emploi de celles-ci est le grand art du gouvernement et le prince, semblable à un dieu, a établi des lois de liberté qui garantissent la protection et l'encouragement à l'honnête industrie de tous les hommes" ( $\mathbb{8} 42$ ). Le rôle du gouvernement doit se borner à protéger la propriété des individus des trois inconvénients inévitables de l'état naturel ( $\$ 127)$; il y manque, en effet, "des lois établies, connues, reçues et approuvées d'un commun consentement" ( $\$ 124)$, "un juge reconnu pourvu de l'autorité pour décider de tous les différends selon la loi établie" ( $\$ 125)$, et enfin "un pouvoir qui soit capable d'appuyer et de soutenir une sentence donnée, et de l'exécuter" (\$126). Le rôle et le sens de la société politique sont d'assurer la conservation de la propriété en fournissant les pouvoirs législatif, judiciaire et exécutif ${ }^{47}$. Tel est le fondement, selon ce roman de formation lui-même fondateur, du libéralisme ${ }^{48}$.

\section{«Le heurt successif sidéralement d'un compte total en formation » Mallarmé}

Le paradigme libéral, disions-nous, tend à judiciariser à l'extrême les rapports entre les hommes. Après nous être ainsi penché pour boire quelque peu aux sources libérales, nous saisissons mieux le conception moderne du droit qui tend à considérer davantage, par nature, l'bomo economicus que l'animal politique. Les rapports politiques entre les hommes sont pensés, d'abord et avant tout, comme rapports de l'homme aux choses car c'est à partir de l'homme comme producteur et propriétaire que l'on entreprendera de reconstruire et de médiatiser les rapports des

47. "Le programme libéral, une fois qu'il est complètement élaboré, fait du droit de propriété, et tend à faire de l'économie en général, le fondement de la vie sociale et politique." P. Manent, op. cit., p. 103. Voir également, parmi une florissante littérature secondaire, K. I. Vaughn, John Locke Economist and Social Scientist, Chicago, Chicago University Press, 1987 et J. Tully, John Locke. Droit naturel et propriété, Paris, PUF, 1992.

48. "Blesser les intérêts d'une classe de citoyens, écrit le disciple de Locke, Adam Smith, quelque légèrement que ce puisse être, sans autre objet que de favoriser ceux de quelque autre classe, c'est une chose évidemment contraire à cette justice, à cette égalité de protection que le souverain doit indistinctement à ses sujets de toutes les classes. "Cité par H. Denis, Histoire de la pensée économique, Paris, PUF, 1971, p. 218. L'abstention de l'État, on le voit, est ici exigée au nom de la justice et de l'égalité. 
hommes entre eux; on spécifiera le mien et le tien, les droits qu'un individu peut revendiquer à l'égard de tel ou tel autre sur telle ou telle chose: les hommes entrent en relation en tant que travailleur et propriétaire, acheteur et vendeur, testateur et héritier, etc. Les règles communes que les hommes élaborent entre eux et auxquelles ils s'assujettissent librement cessent d'être représentées comme autant de composantes identitaires de l'être commun; ce qui est commun n'est plus une composante essentielle, mais simplement un moyen juridico-politique de protéger et de permettre l'épanouissement des désirs dans toute la mesure où ils sont compatibles avec les droits d'autrui.

Dès lors, on peut légitimement croire que la "libéralisation" des marchés n'exprime que l'exarcerbation et la conséquence logique de tendances qui étaient d'ores et déjà inscrites dans les prémisses atomistes; être libre, c'est ne dépendre que de soi, vivre le moins de contraintes possibles, que celles-ci proviennent de l'histoire, de la nature, de la société, de la famille, de l'identité sexuelle, etc. Si, en effet, l'État ne se justifie plus par ce qu'il exprime de la vie civique mais par ce qu'il permet de réaliser, c'està-dire se réduit à un instrument dont la tâche est de faciliter l'épanouissement et la satisfaction des désirs et des besoins de l'individu prépolitique, alors on ne voit plus, à l'instar de Hegel, ce qui viendrait fonder quelque allégeance à l'égard de tel ou tel État. Selon ce paradigme, puisque l'homme est déjà lui-même avant toute insertion dans une communauté, le lamento républicain qui évoque nostalgiquement quelque allégeance à une mémoire collective, à un destin commun, une langue, n'apparaît plus que sous les traits du mythe ou d'une fiction folklorisante ${ }^{49}$.

49. Les nations ne seraient que des "communautés imaginées" selon l'expression de B. Anderson. "[Le] néo-herderisme romantique guette toujours le Québec", comme l'écrit Régine Robin qui, à fiction fiction et demie, rêve du libre choix des identités à la carte. "Il nous faut donc tout dévoyer, tout nous approprier et nous désapproprier" en espérant l'avènement d'une grande littérature borgésienne. Je ne saisis pas comment ce motif littéraire - qui me semble du reste naif du point de vue anthropologique et par la conception qu'il présuppose de la langue - est transposable au niveau politique, l'auteure se gardant de nous en indiquer les voies. Qu'est-ce, par exemple, que s'approprier et se désapproprier la clitoridectomie au Québec? Cela dépasse mon entendement." Voir "L’impossible Québec pluriel: la fascination de "la souche" ", Les frontières de l'identité. Modernité et postmodernité au Québec, sous la dir. de M. Elbaz, A. Fortin et G. Laforest, Québec et Paris, Presses de l'Université Laval et l'Harmattan, 1996, p. 309 et 307. 
Du reste, les théories artificialistes du langage des Hobbes, Locke et Condillac ont largement contribué à nous faire croire que les individus inventent ce langage arbitrairement, qu'ils en sont indépendants; le langage ne constitue pas, ontogénétiquement, un réseau d'interlocution premier qui façonne notre pensée et notre identité $^{50}$. Pis, on soupçonnera d'emblée - c'est l'antienne libérale - que l'insistance sur cette immersion ne signifie au fond que l'enfermement dangereusement romantique de l'individu dans une "seconde nature", un "tribalisme anachronique" opposé à la diversité des conceptions privées du bien.

Cette problématique éclaire les débats politiques et juridiques menés par le Parti Égalité (sic), Alliance Québec ou la philosophie qui anime Cité libre. Ils procèdent du refus de reconnaître aux francophones, au nom d'une égalité absolue des libertés individuelles et d'une conception artificialiste du langage, la légitimité d'un but collectif de survivance lorsqu'il se traduit parfois en une limitation raisonnable du libre choix de la langue d'enseignement ou du travail sous certaines conditions. Rejetant toute acceptation des différences réelles et tout compromis politique réaliste, l'incantation ostentatoire de la primauté du droit masque parfois difficilement la haine du parlementarisme et le refus aveugle de penser les réalités politiques, historiques et démographiques au nom d'un conception procédurale de la justice, de principes faussement neutres. Faussement neutres car, nous le savons, l'édification de la nouvelle nationalité canadienne sous Trudeau, qui se poursuit toujours, visait essentiellement à endiguer le nationalisme francophone à l'aide de trois outils principaux : la Charte de 1982, en tant que levier de la centralisation, institue la primauté du droit et priorise la défense des libertés individuelles; la politique du multiculturalisme, en évacuant le biculturalisme, marginalise la spécificité de la nation francophone dans la "mosaïque canadienne" et déplace l'accent au profit des libertés individuelles; enfin, l'encouragement idéologique et financier, par l'État fédéral, des groupes de pression qui naissent suite aux droits consacrés exacerbe encore davantage le juridisme ${ }^{51}$. Or, lorsqu'on sait que

50. Voir Y. C. Zarka, op. cit., p. 65-85, et C. Taylor, "Le langage et la nature humaine", Liberté des modernes, traduction de P. de Lara, Paris, PUF, 1997, p. 21-66.

51. Voir, sur la mythologie du papisme légal canadien, le brillant article de M. Chevrier, "Le papisme légal", Argument, Sainte-Foy, vol. 1, n 2, 1999, p. 73-92. 
le juge en chef de la Cour suprême, Antonio Lamer, se déclare publiquement libertarien, affirme que "l'État n'a pas droits. Ce sont les individus qui ont des droits" ou encore que "les gouvernements ne sont que des instruments à qui on délègue le pouvoir ${ }^{52}$ ", alors nous mesurons à quel point nous vivons encore de l'impulsion artificialiste du XVII ${ }^{\mathrm{e}}$ siècle.

Mais, nous le savons, il n'y a pas que le concept d'état de nature qui procède de la fiction moderne. Le concept de contrat social procède lui aussi d'une fiction permettant de donner la formule de la constitution d'un État, non d'expliquer sa naissance réelle qui dépend de tout autre chose: des rapports de puissance tels qu'ils se sont historiquement développés. Or, le Canada oblitère délibérement de plus en plus son acte de naissance et son histoire réelle (la conquête coloniale, la volonté d'assimilation des francophones, les accomodements afin de repousser la démocratie américaine, les épisodes racistes, etc.) au profit d'un nouveau contrat social, du rapatriement de la Constitution et de l'adoption de la Charte sans le consentement du Québec, et sans jamais en appeler au peuple canadien. Heureusement, nous vivons dans un État de droit. Ce que l'histoire n'a su faire, à savoir nous donner un vernis honorable, l'abstraction atomisante et contractualiste le fera. Là où le discours de la fiction moderne du politique permettait de penser les fondements, ce récit permet maintenant d'occulter l'histoire et de favoriser l'amnésie. De la fiction instituante à la fiction instituée, en somme. Tout ceci témoigne à l'évidence de notre échec à nous constituer comme communauté politique favorisant l'épanouissement des nations en son sein; au vu de quoi, en deçà de toute reconnaissance réelle des différences, certains magnifient en toute bonne conscience les cultures hybrides, métissées, migrantes, et chapeautent le tout de l'étiquette postmoderne... À quand la culture individuelle, le langage privé?

Au seuil de la pensée politique moderne, la fiction chez Hobbes instaura une mise entre parenthèses du monde qualitatif, de l'histoire réelle, des rapports de force tels qu'il se sont développés, et prit pour objets des êtres abstraits, ces atomes que sont les individus, afin de reconstruire et de fabriquer un être fictif, Léviathan, qui assurerait la conservation de soi. "On peut, par une réflexion industrieuse, découvrir des principes rationnels propres à

52. Le Soleil, Québec, 4 septembre 1999, p. A 18. 
54

faire que la constitution [d'une République] dure à jamais ${ }^{53}$.". Si les lois du marché, cet automate autorégulateur, assurent mieux que les États, qui imposent encore quelques irrationnels contraintes fiscales, sociales, culturelles ou environnementales, le plein épanouisement de l'individu, alors on ne voit pas ce qui empêcherait le propriétaire d'y voir la seule source de légitimité, parce que plus efficace. Si le cadre national - même canadien - fut nécessaire au déploiement de l'économie de marché, il n'apparaît plus désormais, aux yeux de bien des élites économiques et intellectuelles, que sous l'aspect d'une dernière contrainte à abattre au profit d'un individu toujours plus délesté de tout héritage et de tout poids. La fiction hobbesienne de l'individu est devenu notre réalité contemporaine, la fiction lockéenne de l'appropriation illimitée est devenue notre cauchemar. Du coup, l'alternative fondamentale entre l'état de nature et l'état artificialiste se repose à nous sous un jour étrangement nouveau et de façon plus dramatique: qu'est-ce qui est le plus fondamental en l'homme, un désir perpétuel d'acquérir pouvoir après pouvoir, ou le désir rationnel de la conservation de soi? Espérons que ce dernier désir ne se révèle pas fictif.

53. Léviathan, p. 359. 